\title{
BMJ Open Psychosocial impacts on the Christchurch Muslim community following the 15 March terrorist attacks: a mixed-methods study protocol
}

\author{
Ruqayya C. Sulaiman-Hill (D) , ${ }^{1}$ Richard Porter (D) , ${ }^{1}$ Sandila Tanveer (D) , ${ }^{1}$ \\ Joseph Boden (D) , ${ }^{1}$ Ben Beaglehole (D) , ${ }^{1}$ Philip J Schluter (D) , ${ }^{2,3}$ \\ Shaystah Dean (1) , ${ }^{1}$ Caroline Bell (i) ${ }^{1}$
}

To cite: Sulaiman-Hill RC, Porter R, Tanveer S, et al. Psychosocial impacts on the Christchurch Muslim community following the 15 March terrorist attacks: a mixed-methods study protocol. BMJ Open 2021;11:e055413. doi:10.1136/ bmjopen-2021-055413

- Prepublication history and additional supplemental material for this paper are available online. To view these files, please visit the journal online. (http://dx.doi.org/10.1136/ bmjopen-2021-055413).

Received 12 July 2021 Accepted 29 July 2021

Check for updates

(c) Author(s) (or their employer(s)) 2021. Re-use permitted under CC BY-NC. No commercial re-use. See rights and permissions. Published by BMJ.

${ }^{1}$ Department of Psychological Medicine, University of Otago, Christchurch, New Zealand ${ }^{2}$ School of Health Science, University of Canterbury, Christchurch, New Zealand ${ }^{3}$ School of Clinical Medicine, University of Queensland, Brisbane, Queensland, Australia

Correspondence to Dr Ruqayya C. Sulaiman-Hill; ruqayya.sulaiman-hill@otago. ac.nz

\section{ABSTRACT}

Introduction On 15 March 2019, a white supremacist gunman opened fire in two mosques in Christchurch, New Zealand, during Friday prayers, killing 51 people and injuring 40 . The event was witnessed by at least 250 survivors and also live streamed on social media, leading to widespread and repeated exposure within the community. It is expected that survivors, families and community members will be at increased risk of developing mental disorders due to the scale and violence of these attacks.

This protocol describes the first phase of a proposed longitudinal study to screen and assess the longterm impacts of the terrorist attack on members of the Christchurch Muslim community, to determine clinical need and facilitate access to appropriate interventions and to gain insights into working with such a traumatised, ethnically diverse population. It has been developed in close collaboration with members of that community.

Methods and analysis A mixed-method design is described, combining self-report measures with a clinician-administered diagnostic interview. Participants include Christchurch Muslims aged 18 years and over, with a target sample size of $n=200$. Analyses will determine prevalence of major mental disorders, while regression analyses will model the relationship between pre-event features, trauma exposure and mental disorders. A small number of participants, stratified by exposure type, will also take part in a qualitative interview in English. All study information and self-report measures are provided in translations based on the ethnolinguistic composition of the group and are available in paper and online versions.

Ethics and dissemination Ethical approval was granted by the New Zealand Health and Disability Ethics Committee 19/NTA/147. All participants provide informed consent, either written or online via REDCap software. Findings will be disseminated initially to the impacted community, then by publication in scientific journals, presentations and to government agencies.

Trial registration number The study is registered with the Australian New Zealand Clinical Trials Registry, ACTRN12620000909921.

\section{Strengths and limitations of this study}

$\Rightarrow$ The study design was guided from the outset by strong collaboration between the study team, composed of clinical experts and Muslim researchers, the impacted Muslim community and service providers.

$\Rightarrow$ Mixed methodology combining standardised psychometric measures in appropriate languages with a clinical interview for mental health screening provides a comprehensive picture of individual and community impacts.

$\Rightarrow$ A participant-centred approach, reflecting the cultural and linguistic diversity of the group and using ethnically matched research assistants, is critical for working with such a highly traumatised, ethnically diverse community.

$\Rightarrow$ Representativeness is compromised by reluctance of traumatised individuals to participate and entrenched stigma concerns around mental health issues.

$\Rightarrow$ The length and density of the interview does not provide many opportunities for detailed discussion of participant experiences, which can also be cathartic, so a separate qualitative study was set up to capture these rich data.

\section{INTRODUCTION}

On 15 March 2019, a white supremacist gunman opened fire in two mosques in Christchurch, New Zealand ${ }^{1}$ during Friday juma'a (congregational) prayers. The attacks lasted only a few minutes but resulted in 51 fatalities. A further 40 people were shot (including some with serious, life-changing injuries), many others had minor injuries and there were up to 250 survivors. The scale and violence of these attacks is unprecedented in New Zealand ${ }^{1}$ and is one of the worst mass shootings in history. People present in the mosques and their families are expected to be at increased risk of developing mental health 
disorders, a concern acknowledged by those within the small Christchurch Muslim community community of about 4000 people. $^{23}$ This paper describes the development of a study protocol to screen and assess the longterm impacts on this group, all of whom shared a Muslim faith but came from ethnically and socially diverse backgrounds. Recruitment for the project commenced in 2020, but due to COVID-19 impacts, interviewing is expected to continue until 2022.

\section{Mass shootings and their impact on mental health}

Mass shootings and other forms of mass trauma are increasing in frequency worldwide, and in recent years, there have been several attacks with religious and racial motivations. ${ }^{4}$ Most of the evidence on the psychological effects has been reported from the USA, Europe and Scandinavia. Notable incidents in the USA include the killing of six worshippers during prayer at a Sikh temple in $2012,{ }^{56}$ nine killed at a black church in $2015^{7}$ and more recently the shooting of 11 Jewish worshipers at the Tree of Life Synagogue in Pittsburgh in 2018. ${ }^{8}$ Elsewhere, 3 migrant school students were killed in Sweden in 2015, ${ }^{9}$ 6 Muslim worshippers were killed during evening prayers in Canada in $2017^{10}$ and 267 worshippers were killed by bomb attacks during Easter church services in Sri Lanka in April 2019. ${ }^{11}$ One of the worst mass shootings fuelled by anti-immigrant, anti-Muslim sentiment occurred in July 2011 when 77 people, more than half of them teenagers, died in consecutive attacks at Utoya Island and Oslo, Norway. ${ }^{12}$ This was a xenophobic, politically motivated attack inspired by liberal immigration policies, which was directed against the government and labour party youth. ${ }^{4}$

Apart from the Norwegian massacre, the Christchurch shooting is the worst attack deliberately targeting Muslims in a Western context. Those exposed to this incident share a common faith but have a diverse ethnic and social composition, which is different from most previous groups. An important additional contextual factor in the Christchurch attack was the live streaming on social media, resulting in wider and repeated exposure to a demographically and geographically disparate audience, including children and adolescents.

The psychological sequelae of exposure to violence is vast, with symptom trajectories varying over time and among different groups. A recent systematic review ${ }^{13}$ of 49 articles on samples of survivors and members of affected communities in the aftermath of 15 mass shooting incidents identified prevalence estimates of up to $91 \%$ for post-traumatic stress disorder (PTSD) and $71 \%$ for major depression. Individual responses can also include anxiety, grief, anger, sleep problems and somatisation symptoms. Factors known to predict adverse outcomes include demographic and preincident characteristics, such as female gender, preincident psychological symptoms and previous exposure to trauma. Similarly, event exposures, including greater proximity to the attack, acquaintance with the deceased, perception of life being in danger, panic or dissociative responses at the time, are also important. Postevent, risk factors for detrimental outcomes include individual psychological attributes, for example, emotion regulation difficulties, and psychosocial factors such as having less social support, or a change in psychosocial resources. ${ }^{14}$

Although relatively little is known about the longer term psychological and emotional consequences of these experiences on individuals exposed to such events, ${ }^{15}$ potential prolonged and significant impacts have been increasingly noted in the literature, including in affected relatives. ${ }^{1516}$ Similarly, there is developing evidence that communitywide collective trauma can result from large-scale events and that the consequences may be more profound for those from collectivist cultures. ${ }^{17} 18$

Gaps in the extant literature highlight the need for studies in the aftermath of high-impact events to include multiple waves of follow-up data in both exposed populations and those bereaved by the events ${ }^{13}$ to enhance understanding of the longitudinal patterns of responses and the processes that lead to chronic psychological symptoms. It is also desirable to include measures of positive outcomes (eg, resilience and post-traumatic growth $)^{13}$ and, particularly important, when working with diverse populations, for assessments and interventions to be culturally and linguistically appropriate..$^{190}$

\section{Impacts on physical health}

The observations that severe trauma and stress impact aspects of physical health are well established; for example, chronic musculoskeletal pain, hypertension, hyperlipidaemia, obesity and cardiovascular disease have been reported following trauma and stress. ${ }^{21}$ These may be indistinguishable from an increase of somatic symptoms, which are also regularly observed following traumatic stress, and are more commonly reported in non-European ethnic groups. ${ }^{22}$ It is important when assessing the health status of such affected groups to also measure physical symptoms and morbidity to understand the overall impact that this type of trauma has on both physical and psychological well-being.

\section{Cultural considerations}

The nature and context of the Christchurch shootings in the two mosques, specifically targeting an ethnically diverse group of Muslims, highlights the need for cultural sensitivity and better understanding of Muslim beliefs and practices by researchers, clinicians and the wider community. Although experiences of intense emotional distress following traumatic events are likely to be universal, there is evidence to suggest that the expression of that distress is subject to substantial cultural variation. ${ }^{23}$ The 2007 United Nations Inter-Agency Standing Committee Guidelines on Mental Health and Psychosocial Support in Emergency Settings recommends 'tailoring assessment tools to the local context' and investigating the full breadth of psychological disorders and symptoms including cultural conceptions of distress. ${ }^{24}$ Several investigators have suggested that the PTSD diagnosis has limited content 
validity among traumatised members of diverse cultural groups because it does not include somatic symptoms. ${ }^{22}$ It is recommended that rather than solely focusing on diagnostic criteria for PTSD, other health indicators are also included in assessments. ${ }^{25}$ The importance of assessing current and postevent non-traumatic stressors/loss, and past traumas, in both assessment and treatment interventions, ${ }^{1426}$ as well as the importance of using ethnically and culturally appropriate methods and measures, is also emphasised. ${ }^{27}$

The study described here forms the first phase of a proposed longitudinal study to follow the members of the small Christchurch Muslim community who have been most impacted by the terrorist attack on the two mosques in 2019. This paper outlines the development of a study protocol to address these issues and outlines our approach to working with such an ethnically diverse and highly traumatised population.

\section{Research aims}

This research aims to answer the following questions.

- What are the psychological effects of the Christchurch mosque attacks on this highly exposed group of Muslims and their families? It will provide culturally informed insights into the outcomes for these traumatised groups and therefore their needs in the months and years following trauma.

- How can assessment of clinical need be best achieved in this population? The project will test a real-life screening programme that we anticipate will improve detection of significant mental health difficulties in those most affected. In addition to assessing for mental health difficulties, the study aims to provide clear pathways for participants to access appropriate interventions and guide development of culturally competent services.

- What learnings can be obtained from working with such a traumatised, ethnically diverse community? The project will inform evidence regarding the best approach to screening following future mass trauma events, in particular for those of the Muslim faith and from ethnically diverse migrant communities.

\section{METHODS AND ANALYSIS \\ Project team}

- Research team: the research team overseeing the study is composed of psychiatrists, psychologists, a mental health nurse and specialists in international health, public health and longitudinal studies. Some members of the team are from the Muslim community.

- Clinical team: five specialist mental health nurses and a clinical psychologist conduct the clinical interviews, liaise with the psychiatrists on the clinical team and facilitate referrals as needed. Three psychiatrists provide clinical oversight.

- Muslim research assistants (RAs): RAs from the Christchurch Muslim community assist with recruitment, facilitate interviews, help with translating study material and provide language support during interviews if necessary. Almost 30 RAs have been involved with the project since it started. They all speak English and at least one other study language and most have academic, medical/nursing or psychology backgrounds.

\section{Training, support and supervision for the project team}

It is recognised that training and regular supervision are necessary for researchers with indirect exposure to trauma. ${ }^{26}{ }^{28}$ Research staff working in this study hear trauma narratives during the process of data collection, so there is a potential for vicarious trauma or compassion fatigue to develop. This is a particular concern for those already exposed through dual relationship community support roles. RAs and nurses have regular supervision with registered clinical psychologists.

Clinicians, nurses and RAs also meet regularly and participate in training sessions together, discussing topics such as the importance of supervision, cross-cultural learning and any general issues that have arisen. These forums also provide opportunities to debrief.

At the commencement of employment, RAs receive training in the interview process, mental health disorders, telehealth technologies, working as an interpreter, privacy issues, confidentiality and some of the challenges associated with dual relationships when working in small communities where participants may know them socially. The nursing team receive training in the use of the semistructured clinical interview, cultural considerations and working with interpreters.

\section{Patient and public involvement}

Members of the Christchurch Muslim community have been engaged in the project since the outset, providing guidance on the design and implementation of the study, desired outcomes and practical considerations. Muslim leaders working on the response provided endorsement and helpful advice during the development phase. The study reference group was established during the planning phase of the project and continues to provide oversight and input through regular meetings. This group is composed of Muslims from all the affected groups, including bereaved families, injured, witness survivors, male and female, youth and older people, as well as the Imams from the affected mosques. The group use their personal networks to obtain feedback from the wider community, to promote the study and help with recruitment. In due course, they will also provide cultural insights during analysis and help to facilitate dissemination of results. Furthermore, the study has been endorsed by the Federation of Islamic Associations of New Zealand as the preferred one-point approach to establish community needs.

Feedback from engagement with the impacted community revealed that mental health issues are a significant concern, so measures to assess this were seen as important. There was also a desire to highlight some of the positive 
aspects, such as the role of religion in coping and post traumatic growth. The project is seen by the community as an opportunity to gather data on issues of community concern, including experiences of discrimination and other social disparities. The final selection of measures needed to balance the research burden and the length of the interview, against the need for information.

Every stage in the development and implementation of the study has involved close collaboration with Christchurch Muslims. We believe this is critical to the acceptance and success of the project and ongoing recovery initiatives, as concerns around potential research reticence, stigma and interview fatigue have been raised. All research assistants and several members of the research team are local Muslims, so they have a personal interest in ensuring the study delivers the best outcomes for their impacted community.

Collaboration between the research team, clinical services and the wider community

The research questions and selection of outcome measures were largely guided by the previous experience of the team dealing with the psychosocial sequelae of trauma, ${ }^{29}$ international responses to similar incidents and experience working with ethnically diverse former refugee populations, ${ }^{30}$ as well as practical considerations such as the availability of suitable screening instruments. However, the team also developed international collaborations that helped inform development of the study protocol and selection of instruments. These relationships are also expected to eventually facilitate dissemination of the important findings of this research to the wider, international community.

Other important collaborations also underpin the study. The partnership with the Canterbury District Health Board, the organisation charged with planning and purchasing health services to meet the needs of this population, allows a clear and accessible pathway to appropriate and responsive interventions and for these to be culturally informed. There is also considerable integration between the research team and organisations that are engaged in providing psychological and practical support to the community. Other research team members have expertise in longitudinal studies, public health research and trauma psychiatry.

\section{Research design}

Due to the complexity and impacts of trauma in this highly diverse community, a multifaceted approach was required. A mixed-methods design was adopted, using a range of culturally relevant self-report measures, openended questions and a clinician-administered diagnostic assessment. We anticipate that this will be the first phase of a longitudinal study, with the next proposed follow-up at 5 years postattack. Participants include Christchurch Muslims aged 18 years and over, with a target sample of $\mathrm{n}=200$, to allow sufficient power $(80 \%)$ to detect correlations of 0.2 (two tailed $\mathrm{p}=0.05$ ). Analyses will determine
Box 1 Self-report measures

\section{Current social and demographic factors}

$\Rightarrow$ Ethnicity, years in New Zealand, family composition and social demographics (see online supplemental file 1).

$\Rightarrow$ Quality of life (Personal Well-being Index; see online supplemental file 2). ${ }^{4445}$

$\Rightarrow$ Social support (Social Network Index; see online supplemental file 3). ${ }^{46}$

$\Rightarrow$ Perceived discrimination (Perceived Discrimination Scale; see online supplemental file 4). ${ }^{47} 48$

$\Rightarrow$ Work and social adjustment (Work and Social Adjustment Scale; see online supplemental file 5). ${ }^{49}$

\section{Measure of exposure and impacts}

$\Rightarrow$ Whether injured or bereaved and measure of previous exposure to trauma (see online supplemental file 6).

\section{Mental health and well-being measures}

$\Rightarrow$ Psychological distress (Kessler-10; see online supplemental file 7). ${ }^{50}$

$\Rightarrow$ Post Traumatic Stress Disorder (PTSD Check List-5; see online supplemental file 8). ${ }^{51}$

$\Rightarrow$ Somatic symptoms (Somatic Symptom Scale-8; see online supplemental file 9). ${ }^{52}$

$\Rightarrow$ Well-being (World Health Organisation wellbeing scale-5; see online supplemental file 10). ${ }^{53}$

\section{Coping and post-traumatic growth}

$\Rightarrow$ Religious coping scale - Muslim (see online supplemental file 11). ${ }^{5455}$

$\Rightarrow$ Post-traumatic growth (Post Traumatic Growth Inventory; see online supplemental file 12). ${ }^{56}$

\section{COVID-19 psychosocial impacts}

$\Rightarrow$ COVID-19 Psychosocial Impacts Scale (see online supplemental file 13) (unpublished).

prevalence of major mental disorders, while regression analyses will be used to model the relationship between a range of pre-event features, trauma exposure and mental disorders.

\section{Measures}

A number of culturally appropriate, validated self-report measures assess social factors, exposure and impacts, well-being and distress and coping strategies (see box 1). Other measures include open-ended questions to determine the acceptability of the interview process. All measures (in English) can be accessed as online supplemental files $1-14$.

\section{Physical health}

Participants provide consent to use their National Health Index number, a unique person identifier used in the New Zealand health system, which provides access to assess rates and nature of presentation (but not confidential details of specific consultations) to general practice and hospital services. At later follow-up, aspects of cardiovascular and metabolic health will be examined in more detail. 


\section{Semistructured diagnostic clinical interview}

The Mini-International Neuropsychiatric Interview (MINI) semistructured clinical interview ${ }^{31}$ is used to obtain past and current diagnostic information. This is administered by specialist mental health research nurse or clinical psychologist. It will indicate rates of serious mental health disorders and give an estimate of the percentage of people with significant clinical disorders and therefore the likely utility of such a rigorous process in similar situations. As the study population is culturally and linguistically diverse, data from the screening tools will be compared against the MINI, and any important systematic differences will be noted and explored.

\section{Psychological impacts of COVID-19}

Although to date, New Zealand has escaped the worst effects of the global COVID-19 pandemic and is not currently experiencing community transmission and lockdown conditions, the effects of Covid-19 are wide reaching. Many participants are migrants from severely impacted regions where vulnerable family members are at risk. The COVID-19 Psychosocial Impacts Scale (CPIS) was developed to assess the level of stress resulting from these impacts and to evaluate the relative effects of COVID-19 compared with exposure to the 15 March terrorist attacks. The CPIS measure has now been validated, showing good internal consistency and will be reported and made available separately.

\section{Qualitative interview}

Some participants will also be invited to take part in a separate semistructured qualitative interview. Stratified purposive sampling will be used to identify people with varied experiences of the impacts and recovery process. ${ }^{32}$ This method was chosen in order to gain rich data from a cross section of people affected. The aim is to recruit five to six people from the following groups: bereaved, bullet injured, family members, witnesses and support people in the order that they are recruited for the main study to give a total of 25-30 participants. Interviews will be in English and conducted by two members of the clinical team. They will take about 1 hour and will be recorded and transcribed for analysis. The aim is to explore participant experiences in greater detail and to identify resilience factors. In particular, questions will focus on any impacts in functioning within the areas of social connectedness, religion, physical health, emotional health, meaning, beliefs, culture and usual activities of daily living. The effects of the COVID-19 global pandemic on this group will also be examined. The Semistructured interview prompts are available as online supplemental file 14 .

\section{Language}

Study materials are provided in English and in translation in Arabic, Bangla, Farsi, Turkish, Somali and Urdu to allow participants to choose their preferred language (or combination of languages) for the assessment. Some measures have also been translated into Indonesian and
Malay. A small number of instruments were already available in some languages (eg, WHO-5, PCL-5, K-10 and PWI), but the majority needed to be translated. Considering the complexity of the study population and the degree of trauma experienced, ensuring that translations appropriately captured the psychometric meanings in a culturally appropriate way was essential. Rather than using standard blind translation-back translation methods, the language teams worked closely together using parallel translations from the outset, translating, back-translating and comparing notes in an iterative process to ensure conceptual equivalence. ${ }^{33} 34$ The languages offered reflect the demographics of the group affected, especially those most impacted; however, it is anticipated that the majority of interviews will be conducted in English. Dissemination of translations and accompanying data for all instruments will be provided in due course through publications and on the study website (otago.ac.nz/march) for the benefit of other researchers.

One significant challenge has been keeping language materials up to date and consistent; every change in English resulted in multiple translation and website updates that impacted on the preparation time before interviewing could begin, had financial implications and added a considerable logistic administrative burden.

\section{Participants}

All adult members of the Christchurch Muslim community who were in the city around the time of the attacks are eligible to participate (see box 2). Although the primary focus was initially on the most impacted groups, the criteria were expanded to provide better understanding of the spectrum of experiences and impacts across the whole Muslim community. Many of these people were also affected, through their roles in the response, providing support, loss of friends and colleagues, viewing the video and other shared experiences as members of a visible minority group. Participation is restricted to those in Christchurch due to the challenges of accessing appropriate clinical pathways in other locations.

Although ineligible for this study because the screening instruments are designed for adults, it is anticipated that children and youth under 18 years will be invited to participate

\section{Box 2 Participant inclusion criteria}

$\Rightarrow$ Adult Muslim 18 years and over at the time of interview.

$\Rightarrow$ Present in Christchurch during or shortly after the attacks on 15 March 2019.

$\Rightarrow$ Currently living in Christchurch.

$\Rightarrow$ Participants from the following groups are prioritised for interviews: $\Rightarrow$ Witnesses/survivors who were present at or near either mosque during the attacks (approximately 250 people), including those who were bullet injured.

$\Rightarrow$ Close family members of the 51 people who died (shuhada/martyrs), including non-Muslims for this category only.

$\Rightarrow$ Close relatives and spouses of the witness/survivor group. $\Rightarrow$ Close relatives of bereaved spouses. 
in customised age-appropriate screening projects currently under development. In the meantime, parents with concerns about children have the opportunity to discuss this with the nurse during their own interview, and then the clinical team can follow-up any issues identified.

\section{Recruitment}

The study is advertised using posters and pamphlets in multiple languages at mosques, halal outlets, support providers and other public spaces, and postcards are distributed after Friday prayers and at health days and other community gatherings. They are also given to participants to pass on to their friends and family members following an interview. Word of mouth and endorsement by existing participants is particularly helpful as it provides opportunities for people to ask questions and to understand possible benefits for individuals and the wider community. Building on personal relationships, using chain referral (snowball) methods with Muslim RAs as contact initiation points into the various ethnic groups, and assistance from the study reference group, has also been critical for recruitment. Social media apps are a popular way for participants to contact the team, and interviews can be set up using dedicated email and language-specific phone lines. The study website (https://www.otago.ac.nz/march/index. html) provides comprehensive information about the study, how to participate and who to contact.

\section{Procedure}

Participants receive a Participant Information Sheet prior to the interview and can ask questions before confirming their consent. The RA and clinician (specialist mental health research nurse or clinical psychologist) lead the interviews, which are either face-to-face with paper selfreport measures or virtually on Zoom using online questionnaires on Qualtrics (Qualtrics, Provo, Utah, USA). This depends on participant preference and COVID-19 restrictions at the time. If required, interpreter assistance is available. The interview consists of three stages (see table 1) and normally takes between 1.5 and 3 hours, depending on individual needs. It starts with a general focus then moves to more specific measures prior to the clinical assessment. The final questionnaires have a more positive focus to end the interview. Retail vouchers to the value of NZ\$50 are given as a token of appreciation of their participation.

Following the interview, the clinician liaises with one of the psychiatrists to discuss possible support or treatment options. She then discusses these by phone with the participant and any referrals are actioned.

\section{COVID-19 and online interviews}

Although the COVID-19 situation in New Zealand is well controlled, the potential for lockdowns necessitated setting up online options as an alternative to face-to-face interviews. Consent form and personal information can be obtained using REDCap V.9.1.0 electronic data capture software ${ }^{35} 36$ This is hosted at the University of Otago and guided by Health Information Privacy Principles, Privacy
Table 1 Three-stage interview process

\begin{tabular}{|c|c|c|}
\hline & Procedure & Measures \\
\hline $\begin{array}{l}\text { Questionnaire } \\
\text { booklet } 1\end{array}$ & $\begin{array}{l}\text { Participant completes } \\
\text { self-report measures } \\
\text { with assistance from } \\
\text { RA as required. }\end{array}$ & $\begin{array}{l}\text { Social and } \\
\text { demographic data. } \\
\text { Quality of life (PWI). } \\
\text { Social support (SNI). } \\
\text { Somatic symptoms } \\
\text { (SSS-8). } \\
\text { Psychological distress } \\
\text { (K10). } \\
\text { PTSD (PCL5). } \\
\text { Perceived } \\
\text { discrimination (PDS). } \\
\text { Additional } \\
\text { demographic data } \\
\text { including assessment } \\
\text { of exposure and } \\
\text { impacts. }\end{array}$ \\
\hline $\begin{array}{l}\text { Clinical } \\
\text { interview }\end{array}$ & $\begin{array}{l}\text { Nurse/psychologist } \\
\text { administered interview } \\
\text { using Mini-International } \\
\text { Neuropsychiatric } \\
\text { interview (MINI). RA } \\
\text { leaves during the } \\
\text { interview unless } \\
\text { language support is } \\
\text { needed. This allows } \\
\text { the nurse to discuss } \\
\text { sensitive issues such } \\
\text { as alcohol or drug use } \\
\text { and suicidal ideation } \\
\text { with the participant in } \\
\text { private. }\end{array}$ & - MINI. ${ }^{31}$ \\
\hline $\begin{array}{l}\text { Questionnaire } \\
\text { booklet } 2\end{array}$ & $\begin{array}{l}\text { Self-report measures } \\
\text { completed by } \\
\text { participant with RA } \\
\text { present. The measures } \\
\text { have a more positive } \\
\text { focus to end the } \\
\text { interview. }\end{array}$ & $\begin{aligned} & \text { Work and social } \\
& \text { adjustment (WSAS) } \\
& \text { Well-being (WHO5) } \\
& \text { Religious coping (RCS) } \\
& \text { Post-traumatic growth } \\
& \text { (PTGI) } \\
& \text { COVID-19 impacts } \\
& \text { (CPIS). }\end{aligned}$ \\
\hline
\end{tabular}

The self-report questionnaires are listed in the order that they are administered.

CPIS, COVID-19 Psychosocial Impacts Scale; K-10, Kessler-10 Psychological Distress Scale; PCL-5, Post traumatic Stress Checklist 5; PDS, Perceived Discrimination Scale; PTGI, Post traumatic growth index; PWI, Personal Wellbeing Index; RCS, Religious Coping Scale; SNI, Social Network Index; SSS-8, Somatic Symptom Scale 8; WHO5, World Health Organisation Wellbeing scale 5; WSAS, Work and Social Adjustment Scale.

Act and Ethics Committee specifications that require a secure survey tool. ${ }^{37} 38$

A procedure for conducting telehealth interviews over Zoom (V.5.4.7) ${ }^{39}$ was developed. This uses the full function Zoom platform, which offers a complete range of security features, including password protection, requiring the host to be present before the meeting starts, using the waiting room option and providing the ability to lock the meeting once the participant has joined. Zoom interviews are not recorded. These features, along with our telehealth protocol requiring all users to be in a private space, to have a support person available and to wear headphones, help to mitigate ethical concerns around security and confidentiality in the use 
of telecommunication technologies and other external constraints that may affect data collection. ${ }^{40}$

All study materials have been uploaded to the Qualtrics web-based survey application ${ }^{41}$ and can be accessed via an emailed link. Participant responses to these self-report measures (identified only by ID codes) are uploaded directly into the system. Qualtrics provides the most effective means of dealing with multiple languages simultaneously. Once uploaded, the various languages can be scrolled through easily, giving participants the ability to switch between them in real-time while completing the questions.

This online option is now provided as an alternative to face-to-face interviews, depending on the personal preference of the participant, as a way to increase accessibility and provide the convenience of flexible scheduling. The use of online survey platforms provides many advantages for researchers as data are captured automatically within the system, reducing the time required for data entry and helping to eliminate transcription errors. Staff can also access data responses while the participant is still working through other measures, allowing them to evaluate psychological impacts before commencing the clinical interview. However, despite these research advantages, many participants still prefer personal contact with paper questionnaires, at least while COVID-19 restrictions are not in place.

\section{Confidentiality}

Confidentiality is strongly emphasised to ensure individuals feel confident about participating. As this is a significant concern for members of such a small, highly interconnected community, every effort that is taken to mitigate any chance of potential breaches in confidentiality helps to provide reassurance to potential participants. Study materials and data are deidentified, and outcomes of the clinical interview and referrals are confidential within the clinical team.

\section{Data analysis}

The reporting of results will follow current best practice, including utilisation of the Strengthening the Reporting of Observational Studies in Epidemiology guidelines (see: www. strobe-statement.org). ${ }^{42}$ The numbers of people present at the mosques during the shootings is approximately 250 . Therefore, the response rate can be calculated for this group, and an assessment of the representativeness of the group recruited can be determined. However, for the other groups (close family members of the 51 people who died (shuhada/ martyrs), close relatives and spouses of the witness/survivor group and close relatives of bereaved spouses), the numbers are not known. In these cases, although rates of mental disorder can be reported, representativeness cannot be determined. Analysis will, in these cases, focus on prediction of outcomes (logistic regression). The target sample is $n=200$, to allow sufficient power (80\%) to detect correlations of 0.2 (two tailed $\mathrm{p}=0.05$ ).

Initial analyses will determine the prevalence of major mental disorders in the cohort (major depression; anxiety
disorder/PTSD). Logistic regression analyses will be used to predict mental disorders as a function of pre-event characteristics (sex and ethnicity; years in New Zealand; family composition, prior mental health, postmigration difficulties and language proficiency), trauma exposure and peritraumatic stress and postevent social support and resilience. Ordinary least squares regression analyses will be used to predict grief, somatic symptoms, post-traumatic growth as a function of pre-event characteristics, trauma exposure and peritraumatic stress, current mental health, postevent social support, resilience and spirituality.

Rates of attendance at general practice clinics and CDHB services will be compared pre-event and postevent. Rates of mental disorder diagnosed by the semistructured clinical screening interview will be compared with those elicited by the screening questionnaires. Rates of major mental disorder discovered at screening will be compared with rates presenting for treatment to determine the undiagnosed/untreated burden of mental health difficulties.

Participant recruitment and eventual retention to the longitudinal component of the study will be collected and assessed in relation to the target population. Attrition will also be formally assessed (using apposite logistic or binomial generalised estimating equation models) to ascertain whether there are important differential drop-out rates and whether methods (such as multiple imputation) may have utility as a sensitivity analysis.

\section{Qualitative analysis}

Audio recordings from the qualitative interviews will be transcribed and analysed thematically. ${ }^{43}$ This will involve an iterative process of reading and rereading the transcripts to become familiar with the content before initial coding is performed. Codes will then be clustered into related ideas to identify themes. Analysis will be carried out by a small team of experienced qualitative researchers already working on the project, with cultural input from the Muslim research team.

\section{Ethics and dissemination}

Ethical approval was granted by the New Zealand Health and Disability Ethics Committee 19/NTA/147. All participants are required to provide informed consent, either written or online via REDCap. Findings will be disseminated initially to the impacted community, then by publication in scientific journals, presentations and to government agencies.

\section{Registration}

The study is registered with the Australian New Zealand Clinical Trials Registry.

Correction notice This article has been corrected since publication to remove supplementary file 14 , at the request of the authors and the copyright holder.

Acknowledgements We would particularly like to remember the 51 shuhada who lost their lives on 15 March 2019 and acknowledge their family members, and all those who were injured and traumatised by their experiences that day. We would also like to acknowledge members of the reference group, the wider Christchurch Muslim community and everyone else affected by this horrendous tragedy. Thanks 
to Andrea Bartram for development and management of the research database and logistic support for this study.

Contributors CB, RCMS-H and RP conceived the study, and JB, BB, PJS and $\mathrm{SD}$ contributed to the design and study protocol. CB and RCMS-H developed the protocol and selected the measures. ST was responsible for coordinating instrument translation and setting up the online component. RCMS-H drafted the paper and all authors read, critically revised and approved the final version of the manuscript.

Funding The project has been funded by major grants from the Health Research Council of New Zealand (HRC 19/907) and Canterbury Medical Research Foundation (CMRF 01/20). Full peer review process was undertaken by both institutions as part of the grant application process.

Competing interests None declared.

Patient consent for publication Not required.

Provenance and peer review Not commissioned; externally peer reviewed.

Supplemental material This content has been supplied by the author(s). It has not been vetted by BMJ Publishing Group Limited (BMJ) and may not have been peer-reviewed. Any opinions or recommendations discussed are solely those of the author(s) and are not endorsed by BMJ. BMJ disclaims all liability and responsibility arising from any reliance placed on the content. Where the content includes any translated material, BMJ does not warrant the accuracy and reliability of the translations (including but not limited to local regulations, clinical guidelines, terminology, drug names and drug dosages), and is not responsible for any error and/or omissions arising from translation and adaptation or otherwise.

Open access This is an open access article distributed in accordance with the Creative Commons Attribution Non Commercial (CC BY-NC 4.0) license, which permits others to distribute, remix, adapt, build upon this work non-commercially, and license their derivative works on different terms, provided the original work is properly cited, appropriate credit is given, any changes made indicated, and the use is non-commercial. See: http://creativecommons.org/licenses/by-nc/4.0/.

\section{ORCID iDs}

Ruqayya C. Sulaiman-Hill http://orcid.org/0000-0003-0650-1618

Richard Porter http://orcid.org/0000-0002-8695-3966

Sandila Tanveer http://orcid.org/0000-0002-0648-5382

Joseph Boden http://orcid.org/0000-0003-1502-1608

Ben Beaglehole http://orcid.org/0000-0002-9521-1745

Philip J Schluter http://orcid.org/0000-0001-6799-6779

Shaystah Dean http://orcid.org/0000-0001-5060-1611

Caroline Bell http://orcid.org/0000-0003-3423-5169

\section{REFERENCES}

1 Wilson N, Thomson G. Mass shooting in Christchurch and the epidemiology of sudden mass fatality events in New Zealand. N Z Med J 2019;132:68-70.

2 Royal Commission of Inquiry. Ko tō tātou kāinga tēnei report: Royal Commission of inquiry into the terrorist attack on Christchurch masjidain on 15 March 2019, Wellington, NZ, 2020. Available: https:// christchurchattack.royalcommission.nz/

3 Statistics NZ. Census 2018. Available: https://www.stats.govt.nz/ 2018-census/

4 Auger VA. Right-Wing terror: a fifth global wave? Perspect Terror 2020;14:87-97.

5 CNN. Gunman, six others dead at Wisconsin Sikh temple, 2012

6 Wikipedia. Wisconsin Sikh temple shooting, 2012. Available: https:// en.wikipedia.org/wiki/Wisconsin Sikh temple_shooting\#: :text= On\%20August \%205,\%202012,\%20a\%20mass\%20shooting\% 20 took, seventh\%20victim\%20died\%20of\%20his\%20wounds\% 20in\%202020 [Accessed 18 Feb 2020].

7 Horowitz J, Corasaniti N, Southall A. Nine killed in shooting at black church in Charleston, in New York times. New York, 2015.

8 Winter T, Romero D, Smith S. How a deadly shooting unfolded at tree of life Synagogue in Pittsburgh, 2018. Available: https://www. nbcnews.com/news/us-news/tree-life-synagogue-deadly-shootingn925291 [Accessed 18 Feb 2021].

9 BBC. Sweden school killings: Attacker 'had racist motives'; 2015.

10 Global News Canada. Quebec City terrorist attack on mosque kills 6, injures 8. Canada, 2017.

11 CNN. Bombs tear through Sri Lankan churches and hotels, killing 250 people, 2019. Available: https://edition.cnn.com/2019/04/21/ asia/sri-lanka-explosions/index.html
12 The Guardian. Norway attacks: at least 92 killed in Oslo and Utøya island, 2011. Available: https://www.theguardian.com/world/2011/jul/ 23/norway-attacks

13 Lowe SR, Galea S. The mental health consequences of mass shootings. Trauma Violence Abuse 2017;18:62-82.

14 Hobfoll S. Resource caravans and resource caravan passageways: a new paradigm for trauma responding. J Mental Health Psych Support 2014;12:21-32

15 Rowhani-Rahbar A, Zatzick DF, Rivara FP. Long-Lasting consequences of gun violence and mass shootings. JAMA 2019;321:1765-6.

16 Dyregrov K, Dyregrov A, Kristensen P. Traumatic bereavement and terror: the psychosocial impact on parents and siblings 1.5 years after the July 2011 terror Killings in Norway. J Loss Trauma 2015;20:556-76.

17 Ali DA, Figley CR, Tedeschi RG, et al. Shared trauma, resilience, and growth: a roadmap toward transcultural conceptualization. Psychol Trauma 2021. doi:10.1037/tra0001044. [Epub ahead of print: 17 Jun 2021].

18 Somasundaram D. Addressing collective trauma: conceptualisations and interventions. Intervention 2014;12:43-60.

19 Harvard Catalyst. Cultural competence in research. Harvard, 2010.

20 Marshall PA. "Cultural competence" and informed consent in international health research. Camb Q Healthc Ethics 2008;17:206-15.

21 McFarlane AC. The long-term costs of traumatic stress: intertwined physical and psychological consequences. World Psychiatry 2010;9:3-10.

22 Kirmayer LJ, Sartorius N. Cultural models and somatic syndromes. Psychosom Med 2007;69:832-40.

23 Rasmussen A, Keatley E, Joscelyne A. Posttraumatic stress in emergency settings outside North America and Europe: a review of the emic literature. Soc Sci Med 2014;109:44-54.

24 Inter-Agency Standing Committee (IASC). IASC guidelines on mental health and psychosocial support in emergency settings. Geneva: IASC, 2007.

25 Hinton DE, Lewis-Fernández R. The cross-cultural validity of posttraumatic stress disorder: implications for DSM-5. Depress Anxiety 2011;28:783-801.

26 Miller KE, Omidian P, Rasmussen A, et al. Daily stressors, war experiences, and mental health in Afghanistan. Transcult Psychiatry 2008;45:611-38.

27 Ahmer S. Psychiatric rating scales in Urdu. J Pak Psychiatr Soc 2010;7:S1-20.

28 van der Merwe A, Hunt X. Secondary trauma among trauma researchers: lessons from the field. Psychol Trauma 2019;11:10-18.

29 Fergusson DM, Horwood LJ, Boden JM, et al. Impact of a major disaster on the mental health of a well-studied cohort. JAMA Psychiatry 2014;71:1025-31.

30 Sulaiman-Hill CMR, Thompson SC. Sampling challenges in a study examining refugee resettlement. BMC Int Health Hum Rights 2011;11:2.

31 Sheehan DV, Lecrubier Y, Sheehan $\mathrm{KH}$, et al. The mini-international neuropsychiatric interview (M.I.N.I.): the development and validation of a structured diagnostic psychiatric interview for DSM-IV and ICD10. J Clin Psychiatry 1998;59:22-33.

32 Palinkas LA, Horwitz SM, Green CA, et al. Purposeful sampling for qualitative data collection and analysis in mixed method implementation research. Adm Policy Ment Health 2015;42:533-44.

33 Ozolins U, Hale S, Cheng X, et al. Translation and backtranslation methodology in health research - a critique. Expert Rev Pharmacoecon Outcomes Res 2020;20:69-77.

34 Barger B, Nabi R, Hong LY. Standard back-translation procedures may not capture proper emotion concepts: a case study of Chinese disgust terms. Emotion 2010;10:703-11.

35 Harris PA, Taylor R, Minor BL, et al. The REDCap consortium: building an international community of software platform partners. J Biomed Inform 2019;95:103208.

36 Harris PA, Taylor R, Thielke R, et al. Research electronic data capture (REDCap)--a metadata-driven methodology and workflow process for providing translational research informatics support. J Biomed Inform 2009;42:377-81.

37 Office of the Privacy Commissioner. Health information privacy code 2020. Available: https://www.privacy.org.nz/assets/Codesof-Practice-2020/Health-Information-Privacy-Code-2020-websiteversion.pdf

38 Office of the Privacy Commissioner. Telecommunications information privacy code 2020, 2020. Available: https://www.privacy.org.nz/ assets/Codes-of-Practice-2020/Telecommunications-InformationPrivacy-Code-2020-website-version.pdf 
39 Zoom Video Communications, Inc. Zoom version: 5.4.7 (59784.1220). 2012-2020.

40 APA. Guidelines for the practice of telepsychology. American Psychological Association, 2013.

41 Qualtrics. Qualtrics, Provo, UT, USA 2005.

42 von Elm E, Altman DG, Egger M, et al. Strengthening the reporting of observational studies in epidemiology (STROBE) statement: guidelines for reporting observational studies. BMJ 2007;335:806-8.

43 Crowe M, Inder M, Porter R. Conducting qualitative research in mental health: thematic and content analyses. Aust N Z J Psychiatry 2015;49:616-23.

44 Cummins RA, Eckersley R, Pallant $\mathrm{J}$, et al. Developing a national index of subjective wellbeing: the Australian unity wellbeing index. Soc Indic Res 2003;64:159-90.

45 Lau ALD, Cummins RA, Mcpherson W. An investigation into the cross-cultural equivalence of the personal wellbeing index. Soc Indic Res 2005;72:403-30.

46 Berkman LF, Syme SL. Social networks, host resistance, and mortality: a nine-year follow-up study of Alameda County residents. Am J Epidemiol 1979;109:186-204.

47 Noh S, Kaspar V. Perceived discrimination and depression: Moderating effects of coping, acculturation, and ethnic support. Am J Public Health 2003;93:232-8.
48 Williams DR, Yun Yu, Jackson JS, et al. Racial differences in physical and mental health: socio-economic status, stress and discrimination. $J$ Health Psychol 1997;2:335-51.

49 Mundt JC, Marks IM, Shear MK, et al. The work and social adjustment scale: a simple measure of impairment in functioning. $\mathrm{Br}$ $J$ Psychiatry 2002;180:461-4.

50 Kessler RC, Barker PR, Colpe LJ, et al. Screening for serious mental illness in the general population. Arch Gen Psychiatry 2003;60:184-9.

51 Weathers FW, Litz BT, Keane TM, et al. The PTSD checklist for DSM5 (PCL-5) - standard [measurement instrument]. Available: https:// www.ptsd.va.gov/

52 Gierk B, Kohlmann S, Kroenke K, et al. The somatic symptom scale-8 (SSS-8): a brief measure of somatic symptom burden. JAMA Intern Med 2014;174:399-407.

53 Topp CW, Østergaard SD, Søndergaard S, et al. The WHO-5 well-being index: a systematic review of the literature. Psychother Psychosom 2015;84:167-76.

54 Adam Z, Ward C. Stress, religious coping and wellbeing in acculturating muslims. J Muslim Ment Health 2016;10:3-263.

55 Adam ZM. Acculturative stress, religious coping and wellbeing amongst New Zealand Muslims [Master of Science in Cross-Cultural Psychology thesis]. Wellington: Victoria University, 2015.

56 Tedeschi RG, Cann A, Taku K, et al. The posttraumatic growth inventory: a revision integrating existential and spiritual change. J Trauma Stress 2017;30:11-18. 